\title{
Intensity-modulated radiation therapy: a review with a physics perspective
}

\author{
Byungchul Cho, PhD \\ Department of Radiation Oncology, Asan Medical Center, University of Ulsan College of Medicine, Seoul, Korea
}

Intensity-modulated radiation therapy (IMRT) has been considered the most successful development in radiation oncology since the introduction of computed tomography into treatment planning that enabled three-dimensional conformal radiotherapy in 1980s. More than three decades have passed since the concept of inverse planning was first introduced in 1982, and IMRT has become the most important and common modality in radiation therapy. This review will present developments in inverse IMRT treatment planning and IMRT delivery using multileaf collimators, along with the associated key concepts. Other relevant issues and future perspectives are also presented.

Keywords: Intensity-modulated radiation therapy, Three-dimensional conformal radiotherapy, Radiotherapy planning, Quality assurance

\section{Introduction}

Intensity-modulated radiation therapy (IMRT) has been considered the most successful development in radiation oncology since the introduction of computed tomography (CT) into treatment planning [1]. IMRT has become the most commonly used modality for radiation therapy since the revolutionary concept was first invented 35 years ago [2]. Modern linear accelerator (linac) and treatment planning systems provide IMRT as a default option, and the increase in the use of IMRT is a global trend. In a recent survey, the rate of IMRT use in Korea was shown to have steadily increased, although it was still only 23.2\% in 2016 [3]. However, it might have surged to over 50\% in 2017, because since July 2015, the coverage of IMRT by national health insurance (95\% of the total cost) has been expanded to include almost every cancer indication. Nowadays, it is probable that every center performs IMRT for all those patients who could benefit from the treatment.

This review presents developments in inverse IMRT treatment planning and IMRT delivery using multileaf collimators (MLCs). Potential future developments and relevant issues are also discussed.

\section{What is IMRT?}

The introduction of CT into radiation oncology in the 1980s enabled treatment planning based on three-dimensional anatomical information of the tumor and surrounding healthy tissues, thus facilitating the establishment of threedimensional conformal radiotherapy (3D-CRT). The key features of 3D-CRT treatment planning include beam's eye view (BEV)

Received 15 March 2018, Revised 15 March 2018, Accepted 20 March 2018.

Correspondence: Byungchul Cho, PhD, Department of Radiation Oncology, Asan Medical Center, University of Ulsan College of Medicine, 88 Olympic-ro 43-gil, Songpa-gu, Seoul 05505, Korea. Tel: +82-2-3010-4437, Fax: +82-2-3010-6950, E-mail: cho. byungchul@gmail.com

(c) This is an Open Access article distributed under the terms of the Creative Commons Attribution Non-Commercial License (http://creativecommons.org/ licenses/by-nc/4.0/) which permits unrestricted non-commercial use, distribution, and reproduction in any medium, provided the original work is properly cited.

www.e-roj.org 


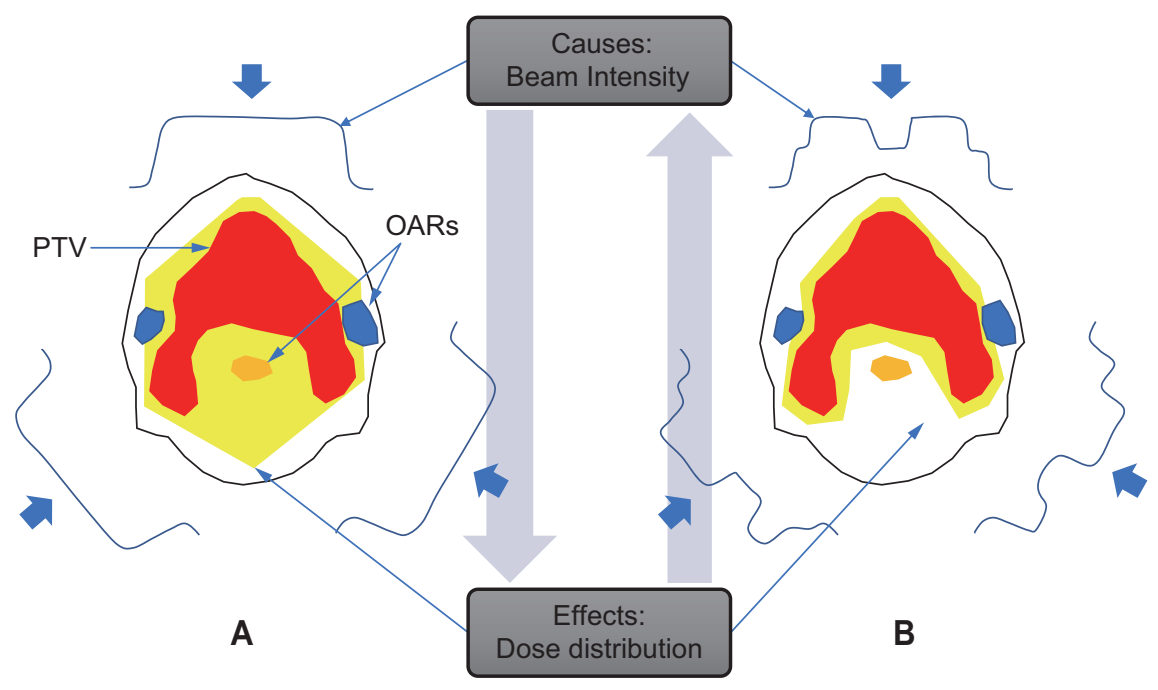

Fig. 1. Comparison of the principle of 3D-CRT (A) and IMRT (B) with illustrations of forward vs. inverse planning. Considering the dose calculation problem of radiation therapy in terms of the concept of causality, while the causes are beam parameters including energy, direction, size, and intensities, the effects are dose distributions. Therefore, conventional planning, in which the beam parameters are given first and the dose distributions are calculated, is 'forward' planning. By contrast, intensity-modulated radiation therapy planning, in which the beam intensities are calculated to provide the given objectives and constraints on dose distributions to the target volume and organs at risk (OARs), is termed 'inverse' planning. 3D-CRT, three-dimensional conformal radiotherapy; IMRT, intensity-modulated radiation therapy; PTV, planning target volume.

design of treatment fields and plan evaluation. BEV allowed for finding a beam direction that could irradiate the tumor without the beam passing through nearby critical organs. Dose-volume histograms (DVHs) and isodose distributions became essential tools for plan evaluation. Together with the progress in $3 \mathrm{D}$ image processing, the $3 \mathrm{D}$ volume information from CT also enabled accurate dose calculation using the convolution-superposition method, allowing the inhomogeneous distribution of tissues to be more accurately handled $[4,5]$.

Whereas 3D-CRT exploits field shape conformation to improve target dose conformality, the organs at risk (OARs) located in the groove region of a concave target volumes cannot be saved from the target dose, as shown in Fig. 1. In conventional 3D-CRT, the irradiation field shape coincides with the shape of the target according to the incidence direction of the irradiation beam, while in IMRT, the beam intensity is modulated according to the arrangement of the target and surrounding organs. The intensities of the rays that pass through OARs are reduced, while the intensities of the rays go primarily through the target volume are increased. The inhomogeneity caused by the 'intentionally non-uniform intensity' of a beam is compensated for by beams from other directions. Physically, a feature of the IMRT technique is to enhance control over the 3D dose distribution through the superposition of a large number of independent segmented fields, either from a number of fixed directions or from directions distributed on one or more arcs. By this method of adding intensity modulation to geometric shaping, the IMRT dose distribution can be rendered concave, as opposed to the convex-shaped coverage accomplished with 3D-CRT, where geometric conformal shaping of a uniform intensity beam is performed. Therefore, IMRT can enable dose reduction to OARs located within a concave area of the planning target volume (PTV).

The minimum unit of the irradiation area that can be individually controlled is called a beamlet. The intensity distribution of the beamlets in an irradiation field is referred to as an intensity map. For example, in the case of a $10 \mathrm{~cm}$ $\times 10 \mathrm{~cm}$ sized field, intensity modulation may be possible for 100 different beamlets of $1 \mathrm{~cm} \times 1 \mathrm{~cm}$, and in the case of a 5 -field treatment, this sum reaches 500 beamlets for which the intensity need to be individually assigned. As it is infeasible for treatment planners, computer optimization is necessary. The dose distribution for the target tumor and OARs are first assigned by the treatment planner, and then the optimization method determines the intensity of the irradiation beam. This is called 'inverse' planning, to distinguish it from 'forward' 
Table 1. Major milestones in the history of IMRT

\begin{tabular}{|c|c|c|}
\hline Year & Investigators & Milestone \\
\hline 1982 & Brahme et al. [2] & $\begin{array}{l}\text { Solving of an 'inverse' problem of rotational beam fluence to deliver a uniform dose } \\
\text { to a donut-shaped target }\end{array}$ \\
\hline 1984 & Brahme [15] & First commercial MLCs patented by Brahme and commercialized by Scanditronix \\
\hline 1988 & Brahme [8] & First paper on algebraic inverse planning \\
\hline 1989 & Webb [9] & $\begin{array}{l}\text { Cast the inverse planning problem as an optimization problem that minimizes an } \\
\text { objective or 'cost' function }\end{array}$ \\
\hline 1990 & Bortfeld et al. [10] & Developed algebraic/iterative optimization for inverse planning \\
\hline 1991 & Boyer et al. [48] & Developed principle of multiple segmented-field IMRT \\
\hline 1992 & Convey and Rosenbloom [18] & Developed principle of sliding-window technique IMRT \\
\hline 1992 & Carol $[16]$ & $\begin{array}{l}\text { First full IMRT system, based on serial tomotherapy, was introduced. NOMOS MIMiC } \\
\text { binary MLC and simulated annealing optimization inverse planning (Peacock Plan) }\end{array}$ \\
\hline 1993 & Mackie et al. [17] & Concept of helical tomotherapy \\
\hline 1994 & $\begin{array}{l}\text { Svensson et al. [19] } \\
\text { Spirou and Chui [20] } \\
\text { Stein et al. [21] }\end{array}$ & $\begin{array}{l}\text { Independent finding of optimal and analytic solutions for sliding-window leaf tra- } \\
\text { jectory problem by three research groups (Karolinska group in Stockholm, MSKCC } \\
\text { group in New York, and DKFZ group in Heidelberg) }\end{array}$ \\
\hline 1994 & Bortfeld et al. [49] & First multiple-static-field (MSF) experiments \\
\hline 1994 & Carol et al. [50] & First IMRT treatment using serial tomotherapy (NOMOS MIMic) \\
\hline 1995 & Ling et al. [11] & First MLC-based IMRT at the Memorial Sloan Kettering Cancer Center \\
\hline 1995 & $Y u[28]$ & $\begin{array}{l}\text { Intensity-modulated arc therapy; proposed as a tomotherapy-mimicking IMRT } \\
\text { using a linac }\end{array}$ \\
\hline 2002 & Park et al. [23] & First IMRT treatment in Korea \\
\hline 2002 & - & Tomotherapy released commercially \\
\hline 2004 & - & $\begin{array}{l}\text { The 14th International Conference on the Use of Computers in Radiation Therapy, } \\
\text { Seoul, Korea, May 9-14, } 2004\end{array}$ \\
\hline 2007 & Otto $[26]$ & Volumetric-modulated arc therapy: IMRT in a single gantry arc \\
\hline 2010 & Bogdanich [38] & New York Times IMRT accident reports \\
\hline
\end{tabular}

IMRT, intensity-modulated radiation therapy; MLC, multileaf collimator.

planning (Fig. 1). Considering the dose calculation problem of radiation therapy in terms of the concept of causality, the causes are irradiation beam and associated parameters including energy, direction, distance, size, and intensity, while the effects are dose distributions. Therefore, the conventional plan, in which the dose distributions are calculated from the given beam parameters, is termed a 'forward' plan. By contrast, the IMRT plan, in which dose distributions (the effects) are given first, and the beam intensities (the causes) are then calculated, is termed an 'inverse' plan.

\section{Development of IMRT}

As IMRT is already a mature technology, a number of excellent historical reviews have been published from various perspectives $[1,6,7]$. Here, a brief history and major milestones are summarized, with a focus on the development of inverse planning and delivery techniques using MLCs (Table 1).

\section{IMRT planning: inverse planning}

In 1982, the paper by Brahme et al. [2] from the Karolinska Institute in Stockholm is generally considered as the first IMRT paper. It presented a solution for beam intensity for rotation therapy that could deliver a uniform dose to a donut-shaped target. Unlike the previous conventional so-called 'forward' approaches, where first the beam intensity is defined and then the dose is calculated, the problem was formulated as an 'inverse' problem, i.e., the desired dose distribution was first defined, and then an integral equation was solved to find an appropriate beam intensity to provide it. We nowadays refer to this process as 'inverse' planning. As a general solution for deriving the optimum incident beam fluence distributions to provide the desired dose distribution to the target volume, Brahme [8] suggested a deconvolution approach that first decomposed or 'deconvolved' the desired dose distribution in the patient into a point-spread dose kernel and point irradiation distributions, and then back projected the point irradiation distributions into fluence profiles. However, 

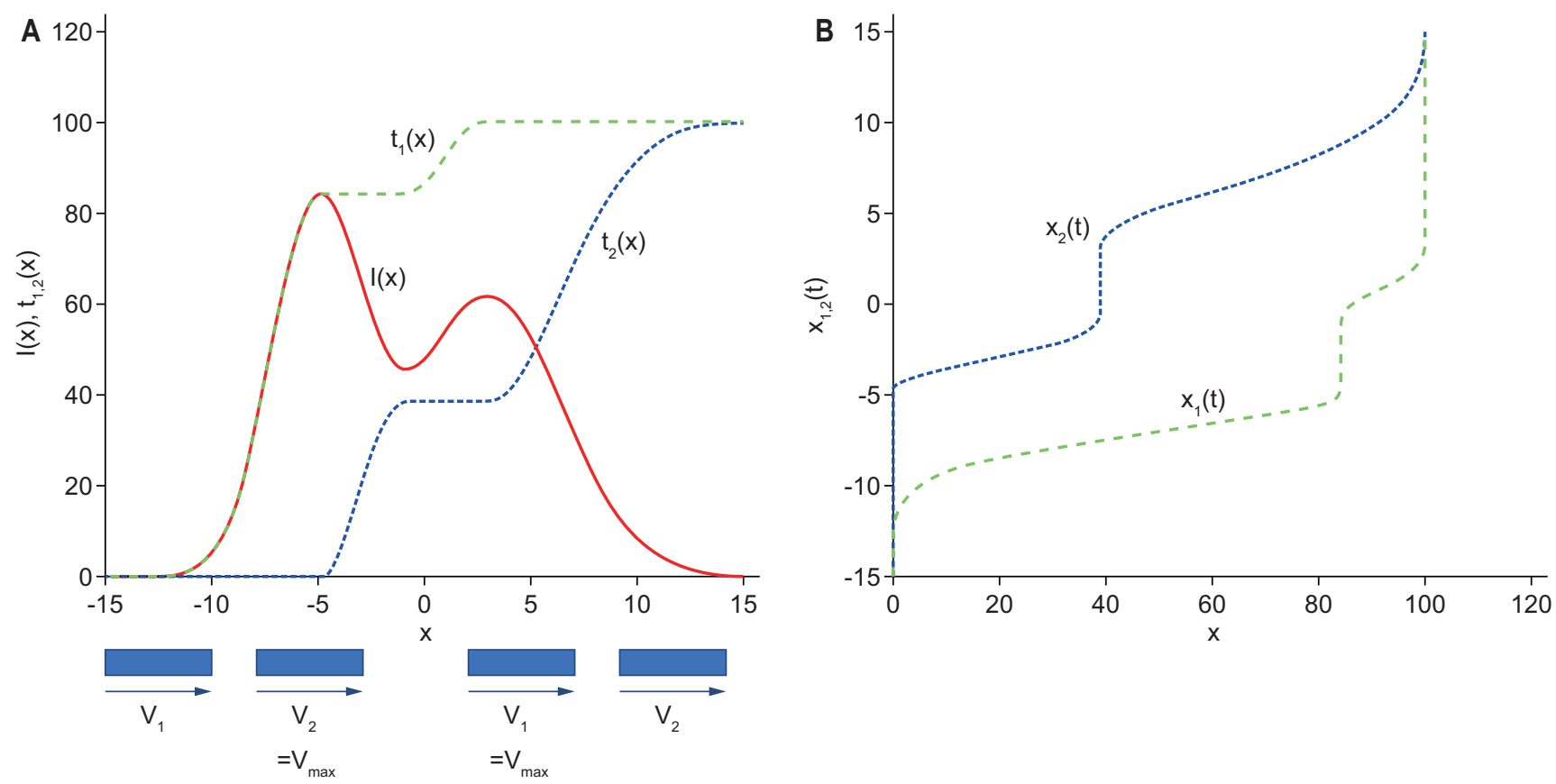

Fig. 2. (A) Multileaf collimator (MLC) leaf trajectories of the trailing leaf \#1 (green dashes) and the leading leaf \#2 (blue dots) as a function of position $x_{1} t_{1}(x)$, and $t_{2}(x)$, according to the intensity profile $I(x)$ (solid red). While the intensity is increasing, the leading leaf \#2 moves at the maximum leaf speed $V_{\max }$ and the trailing leaf \# 1 slows down according to the increasing rate of the intensity. (B) By contrast, while the intensity is decreasing, the trailing leaf \# 1 moves at the maximum leaf speed $V_{\max }$ and the leading leaf \#2 slows down according to the decreasing rate of the intensity.

the process of projection and back-projection had some conceptual drawbacks, in that it could generate negative fluences, and therefore some form of truncation to zero was required; this approach has not been used clinically.

Webb [9] was the first to cast the inverse problem of IMRT as an optimization problem that minimizes an objective or 'cost' function. As there is no exact solution to the inverse problem, the IMRT planning is a process of tradeoffs between target coverage and dose saving to surrounding organs. This concept has become the basic principle of IMRT planning. Bortfeld et al. [10] realized that IMRT planning is a reverse of the process of $\mathrm{CT}$ reconstruction, and that this problem posed as a quadratic objective function does not have local minima, and therefore fast gradient descent methods can be used to find its solution. Several years later this method was employed in the Memorial Sloan Kettering Cancer Center (MSKCC) planning environment, and was used for the first MLC-based IMRT of a prostate cancer patient in 1995 [11].

However, it was eventually recognized that optimization based on simple dose objectives and constraints such as minimum and maximum doses did not yield satisfactory results, and dose-volume limits were introduced for further improvements [12]. Today, dose-volume-based objectives/ constraints are essential in most commercial inverse planning systems. In addition, IMRT optimization based on biological objectives and constraints has been suggested [13]. Another optimization approach in-between pure physical dose objectives (or constraints) and biological planning objectives is effective uniform dose (EUD)-based inverse planning. EUD is the dose that, when homogeneously given to an organ or structure, yields the same biological/clinical effect as a given non-uniform dose distribution [14].

\section{IMRT delivery using a MLC}

The MLC is a device consisting of many individual 'leaves' of a high atomic numbered material, usually tungsten, which can move independently in and out of the radiation beam path to block it. It was originally developed for field shaping in 3D-CRT. Each leaf can be controlled independently and can thereby create a field opening shaped to conform to the projected target volume. The first commercial MLC patented by Brahme [15] was realized by Scanditronics in 1984. MLCs began to become commercially available from major linac manufacturers in the early 1990s. 

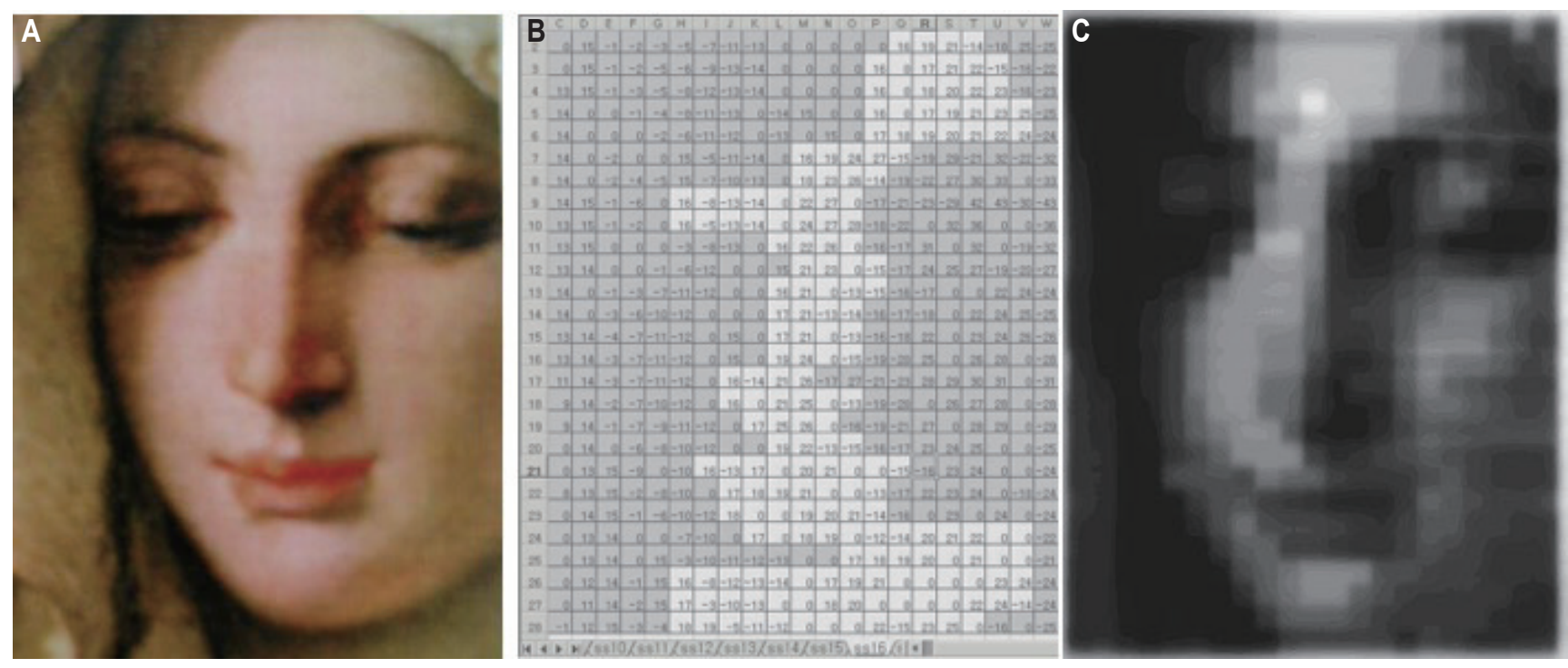

Fig. 3. Renaissance painting of a woman reproduced on X-ray film by the intensity-modulated radiation therapy system. (A) A woman's face from 'Madonna of the Goldfinch' painted by Tiepolo in the Renaissance era, (B) spreadsheet-based calculation of the multisegmental fields, and (C) X-ray film onto which the intensity-modulated beam was delivered.

However, the first apparatus for IMRT delivery was a simple binary-type MLC that was developed for tomotherapy, in which the radiation is delivered slice-by-slice using rotational delivery of a fan beam in the manner of a CT scanner [16]. This was first implemented in the NOMOS MIMic, which delivers rotational IMRT beams in a serial fashion. This was the first commercial system for planning and delivering IMRT, with the first patient being treated in April 1994. Until around the year 2000, the MIMiC was the most commonly used clinical IMRT system in clinical practice. In 1993, Mackie et al. [17] proposed the concept of a rotational delivery of IMRT, where the couch and gantry are in continuous motion akin to a helical CT scanner. Later, this helical tomotherapy (in comparison with the serial tomotherapy of MIMiC) was commercialized in 2002, and went on to replace the MIMiC.

In linac-based IMRT using a MLC, various forms of intensity modulation can be achieved by the movement of a motordriven MLC under computer control. Most IMRT planning systems produce beam intensity maps via an optimization process. These intensity maps are then used in a process called 'leaf sequencing', in which an algorithm attempts to define the shapes (for either Static MLC-IMRT, i.e., 'step-andshoot', or 'field-in-field' mode involving the sequential delivery of radiation subfields that combine to deliver the desired fluence distribution) or trajectories (for Dynamic MLC-IMRT, i.e., the 'sliding-window' mode where the leaves move while the radiation is on) of the MLC leaves required to create a deliverable intensity distribution as close as possible to the distributions calculated by the optimization system.

In 1992, Convery and Rosenbloom [18] developed an outstanding 'leaf sequencing' approach that allowed arbitrary intensity profiles to be produced by a unidirectional 'sweep' motion of the MLC leaves from one side to the other. The proposed method was later used for the first MLC-based IMRT treatment [11]. Three research groups around world: the Karolinska group in Stockholm [19], the MSKCC group in New York [20], and the DKFZ group in Heidelberg [21], independently found almost the same simple analytical solution of the leaf trajectory problem, as originally defined and numerically solved by Convery and Rosenbloom [18], which is illustrated in Fig. 2. The best possible solution can be achieved if the tailing leaf (\#1) shapes those parts of the intensity profile with a positive slope, and the leading leaf (\#2) takes care of the negative slopes. Depending on the velocity profiles of the two leaves, the primary radiation delivered to each point along the path can be made to vary. This finding allows one to create the $t_{1}(x)$ and $t_{2}(x)$ trajectories easily and directly from the desired intensity profile. The leaf motion profiles $x_{1}(t)$ and $x_{2}(t)$ are then obtained by inverting $t_{1}(x)$ and $t_{2}(x)$, respectively (Fig. $2 B$ ).

\section{First IMRT in Korea}

IMRT became a hot topic in Korea at the turn of the 21st century. In August 2000, a new Siemens Primus linac equipped with a double-focused 54-leaf MLC and supporting segmental 


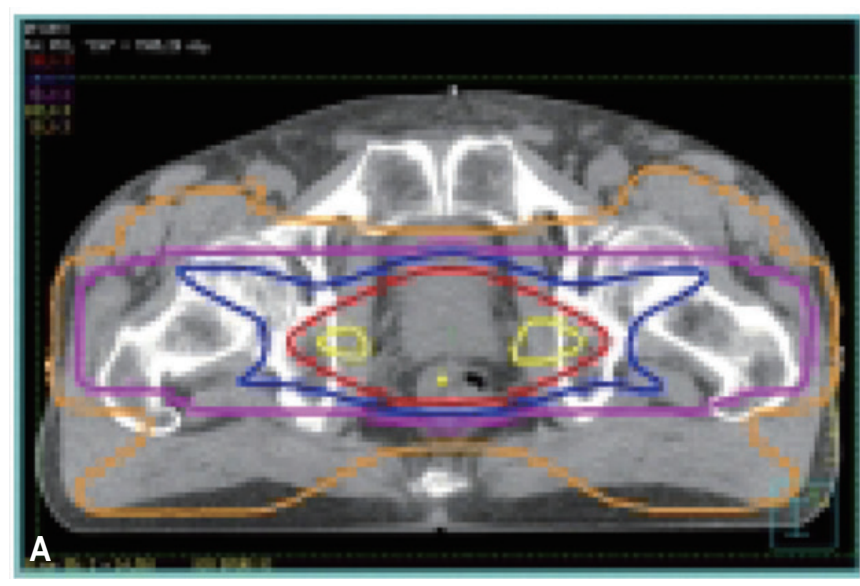

3D-CRT

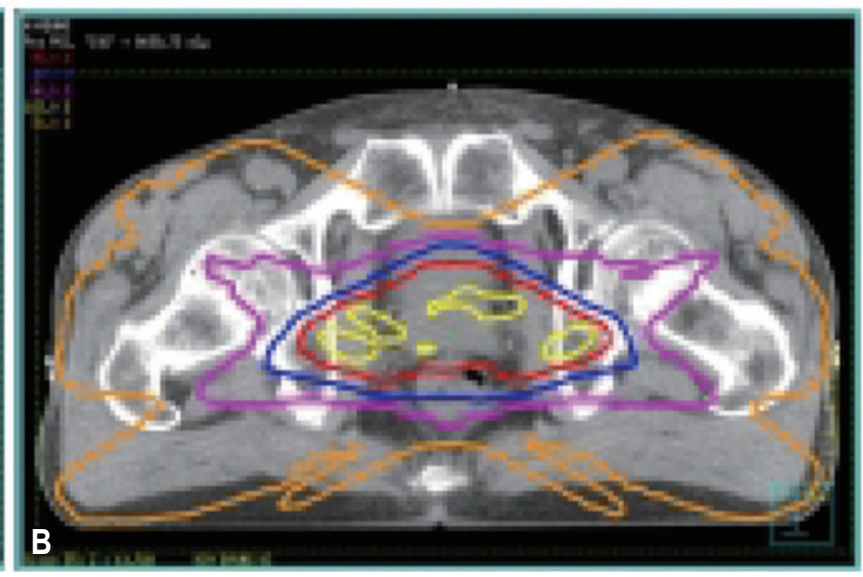

IMRT

Fig. 4. Comparison of isodose curves for 3D-CRT and IMRT plans for the first Korean IMRT treatment of a patient in February 2002. A 76-year-old prostate cancer patient was initially treated with a 3D-CRT plan of 48 Gy in 24 fractions (A), which was then boosted up to 76 Gy with an IMRT plan (B) [23]. 3D-CRT, three-dimensional conformal radiotherapy; IMRT, intensity-modulated radiation therapy.

IMRT was installed at the Hallym University Hospital, and the feasibility of an intensity-modulated beam using 'spreadsheet' calculated multi-segmental fields was demonstrated. As a test piece, a painting of a woman from the Renaissance period was reproduced on X-ray film by the IMRT system (Fig. 3).

After the setting up and commissioning of the Siemens Primus linac, a Pinnacle3 treatment planning system with IMRT optimization (P3IMRT; ADAC Laboratory, Milpitas, CA, USA), and necessary $\mathrm{OA}$ procedures [22], the first Korean prostate cancer treatment was successfully performed on a patient in February 2002 [23] (Fig. 4).

In 2004, a conference contributing to the development of IMRT, the 14th International Conference on the Use of Computers in Radiation Therapy, was held in Seoul, Korea. Many renowned pioneers in the development of IMRT, including Drs. Brahme, Webb, Boyer, and Bortfeld, attended this meeting, and afterwards the use of IMRT quickly spread throughout Korea.

\section{Direct aperture optimization}

In beamlet-based inverse planning, the optimization provides a distribution of the beamlet weights, also called an intensity map. After the optimization is complete, each intensity map is sequenced into a set of deliverable aperture shapes. By contrast, in aperture-based inverse planning, a set of deliverable apertures are included in the optimization, and the optimization parameters include aperture shapes, weights, or both. The optimized treatment plan does not require leaf sequencing, and thus is ready to deliver. Direct aperture optimization (DAO), which directly models the complex segment shapes and their corresponding beam-on time, was first adopted for step-and-shoot IMRT, and was solved with a simulated annealing optimization scheme [24]. After applying speed constraints for gantry rotation and MLC leaf motion, DAO provided a robust solution for intensity-modulated arc therapy (IMAT) [25]. Utilizing the freedom of the linac gantry rotation, arc therapy features much shorter treatment times than static IMRT, and single-arc delivery may be achieved by integrating progressive sampling during optimization [26].

\section{Volumetric-modulated arc therapy}

With the introduction of helical tomotherapy, many people believed that it would ultimately replace linac-based IMRT [27] as it provided highly conformal dose distributions. In this spirit, a tomotherapy-mimicking IMRT using linac IMAT was proposed by $\mathrm{Yu}$ [28]. IMAT is a rotational IMRT that can be delivered using conventional linacs with conventional MLCs. The radiation is on while the gantry is rotating with the MLC leaves moving continuously. Intensity modulation is created by overlapping arcs. This method has been improved with the addition of variable gantry rotation speeds and dose rates, and was introduced as volumetric-modulated arc therapy (VMAT) in 2007 to describe rotational IMRT delivered in a 'single arc' [26]. VMAT can provide highly conformal dose distributions and can significantly improve the IMRT delivery efficiency. The faster treatments reduce the effects of intra-fractional motion on both tumors and organs, and of course, the shorter treatment times also increase patient throughput. The high 
plan quality and fast treatment delivery of VMAT are attractive, and it has been widely applied to many disease sites.

\section{Clinical Perspectives}

\section{Dose prescription, planning, and reporting in IMRT}

With the widespread use of IMRT, substantial variation in the prescribed and delivered doses exists across medical institutions, raising concerns about the validity of comparisons of clinical outcomes of IMRT [29]. Therefore, the isocenter dose in IMRT, which was recommended by the International Commission on Radiation Units and Measurements (ICRU) Reports 50 and 62 for 3D-CRT, is simply a point dose, and often does not reflect the prescription dose that is specified by a selected isodose line encompassing the target volume. ICRU Report 83 [30] recommends that the use of DVHs for the specification of absorbed dose is inherent to IMRT.

\section{Controversial issue: larger skin dose}

The increased numbers of beams and larger Monitor units of IMRT have raised concerns over the higher skin dose compared with 3D-CRT. However, a study on the acute skin toxicity in the breast IMRT revealed that IMRT was associated with a decrease in the severity of acute desquamation compared with a matched control group treated with conventional radiation therapy [31]. To the contrary, another study investigated the cause of acute skin toxicity observed in the treatment of headand-neck cancer. The average dose increase was about 18\%, owing to the bolus effect of the mask. Multiple tangential fields used in the IMRT plans contributed to increases in skin dose of about 19\% and 27\%, with and without the mask, respectively. When the skin of the neck was contoured as a sensitive structure for dose optimization, the volume of skin that received $>45$ Gy was further reduced by about 20\% [32].

\section{Controversial issue: increasing risk of radiation- induced secondary cancer}

Another concern over IMRT is the increasing risk of radiationinduced secondary cancer. Compared with 3D-CRT, IMRT involves more fields, and thus a larger volume of normal tissue is exposed to lower doses. In addition, the number of monitor units is increased by a factor of 2-5, increasing the total body exposure due to leakage radiation. Both factors will tend to increase the risk of secondary cancers. In theory, IMRT is likely to almost double the incidence of secondary malignancies compared with conventional radiotherapy, from about 1\% to $1.75 \%$ for patients surviving 10 years [33]. However, a recent study based on Surveillance, Epidemiology, and End Results (SEER) data for head-and-neck cancer patients between 1992 and 2012 revealed that a comparison of the 3D-CRT period (1992-2009) with the IMRT usage period (2010-2012) showed that the annual incidence rate of secondary malignancy remained consistently below that of patients who received no radiation, indicating no evidence of an increase in secondary cancer [34]. In addition, another SEER data study for secondary cancer rates in 39,028 men with non-metastatic prostate cancer treated with IMRT vs. 3D-CRT [35] showed that (1) there was no difference in the risk of leukemia or myelodysplasia after IMRT compared with 3D-CRT and (2) the risks of colon cancer and rectal cancer were significantly lower after IMRT.

\section{Controversial issue: cost-effectiveness of IMRT}

As mentioned earlier, since 2017, Korean National Health Insurance has covered 95\% of IMRT costs for almost all cancers, and its use is expected to surge upward. There is still some controversy over the cost-effectiveness of IMRT, because it is around twice as expensive as 3D-CRT. In their secondary analysis of the NRG Oncology Radiation Therapy Oncology Group 0617 study (which compared outcomes between patients treated with IMRT vs. 3D-CRT for locally advanced non-small-cell lung cancer [NSCLC]), Chun et al. [36] found no differences in survival, but there was a significantly lower rate of Grade 3 pneumonitis, and therefore recommended that IMRT should be used routinely for locally advanced NSCLC. Clinical evidence of the advantages of IMRT over 3D-CRT is still required, and this is still an on-going research topic.

\section{Quality and Safety in IMRT}

For reasons of accrediting institutions participating in the multi-institutional IMRT clinical trials of the National Cancer Institute and cooperative groups, the Radiological Physics Center (RPC) performed IMRT OA checks in 2008 that revealed that roughly $30 \%$ of institutions failed to deliver a dose distribution to a head-and-neck phantom that agreed with their own treatment plan to within $7 \%$ or $4 \mathrm{~mm}$ [37]. This was surprising, as most institutes were academic hospitals and the tolerance posed was very loose. Quality goes together with safety, and the New York Times [38] reported on serious accidents involving IMRT. This triggered safety concerns in respect to new technologies in radiation therapy, and resulted in safety-related activities from many international organizations. Major contributing factors to adverse radiotherapy incidents are the introduction of new 


\section{Byungchul Cho}

technologies and the increased level of computerization in the radiotherapy treatment workflow [39]. IMRT is time and resource intensive, and environmental and technical concerns need to be addressed to improve patient safety. Timely patient treatment is important, but undue pressure and realtime changes to the treatment plan can lead to errors. The American Society for Radiation Oncology (ASTRO) white paper [40] suggests the use of a 'forced time out', to assure adequate time to perform reviews and QA at key points in the process.

In addition, there are also a few global resources available that allow willing individuals and departments to participate voluntarily and/or anonymously in radiotherapy incident learning systems. These include the International Atomic Energy Agency's Safety in Radiation Oncology (SAFRON) [41], and the recently introduced Radiation Oncology Incident Learning System (RO-ILS) [42] sponsored by ASTRO and the American Association of Physicists in Medicine (AAPM).

As for patient-specific IMRT QA, measurement-based methods are widely used, and are the core element of most IMRT QA programs. In many centers, a QA measurement is routinely performed after a patient's plan is approved by the radiation oncologist. The treatment plan, consisting of MLC leaf sequence files as a function of gantry angle and Monitor units from the patient's plan, is computed on a homogeneous phantom to calculate the dose in the $\mathrm{QA}$ measurement geometry. The delivered doses (point and/or plane distributions) are then compared with the corresponding computed doses. As patient-specific IMRT QA has considerable time requirements, there have been several efforts to streamline the IMRT QA process by introducing independent computer calculation programs to verify the absolute dose at a single point, and the 2D dose distribution from IMRT treatment plans [43]. One advantage of using an independent program for IMRT verification is the rapid movement through the QA process [44]. However, the relative value units (RVUs; a measure of value used in the Korea healthcare reimbursement formula for physician services) of IMRT treatment planning have already been determined with the inclusion of an IMRT $\mathrm{QA}$ measurement. Therefore, pre-treatment $\mathrm{QA}$ seems likely to be performed using a direct measurement for a while longer, as in its current form.

\section{Future Perspectives}

\section{Multi-criterial optimization}

IMRT planning is a multi-criterial optimization (MCO) problem. It always involves tradeoffs, and one can never satisfy the
Radiation Oncology Journal ROJ

perfect dose coverage of the target tumor and the perfect dose saving to OARs, i.e., optimize all the criteria at the same time. A treatment plan cannot be scored with a single 'grade'. An 'optimal' treatment plan is a plan in which we cannot improve one aspect (e.g., reducing the dose in one OAR) without compromising at least one other aspect (e.g., reducing the target dose). Special attention is given to the technique known as Pareto surface navigation, which allows physicians and treatment planners to interactively navigate through treatment planning options to obtain an understanding of the tradeoffs (dose to the target vs. over-dosing of important nearby organs) involved in each patient's plan [45].

\section{Dose painting}

Ling et al. [46] proposed dose painting of the target volume based on biological imaging and biological conformality, with IMRT being an ideal tool to deliver intentionally non-uniform dose distributions for biological conformality. This has not yet been fulfilled because of a lack of sufficient understanding of functional or molecular imaging. However, another form of dose painting is feasible, i.e., functional avoidance radiation therapy. One example is hippocampal sparing radiation therapy for brain tumors, which is helpful for minimizing memory loss. Numerous studies support the feasibility of using modern radiotherapy systems for hippocampal sparing during brain irradiation, including a study of hippocampal sparing whole brain radiotherapy (WBRT) with simultaneous integrated boost (SIB) [47].

\section{Conclusion}

IMRT has been a great success since its inception in the early 1980s and has become the standard clinical modality in radiation therapy. As a physicist, I think it symbolically demonstrates how, in the role of physicists, we can make a major impact on medicine by working together with mathematicians, computer scientists, and engineers. The clinical success of IMRT is expected to continue to be demonstrated with the accumulation of clinical evidence on the advantages of IMRT. I believe that IMRT is now a mature technology, but one that continues to evolve. Therefore, it still remains an interesting area of research and development.

\section{Conflict of Interest}

No potential conflict of interest relevant to this article was reported. 


\section{Acknowledgments}

This work was supported by a National Research Foundation of Korea grant funded by the Korea government (MSIP) (No. 2013M2A2A7043506).

\section{References}

1. Bortfeld T. IMRT: a review and preview. Phys Med Biol 2006;51:R363-79.

2. Brahme $A$, Roos JE, Lax I. Solution of an integral equation encountered in rotation therapy. Phys Med Biol 1982;27:1221-9.

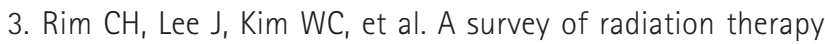
utilization in Korea from 2010 to 2016: focusing on use of intensity-modulated radiation therapy. J Korean Med Sci 2018;33:e67.

4. Mackie TR, Scrimger JW, Battista JJ, Elkhatib E. A convolution method for calculating dose in situations of lateral electronic disequilibrium. Medical Phys 1984;11:397.

5. Ahnesjo A. Collapsed cone convolution of radiant energy for photon dose calculation in heterogeneous media. Med Phys 1989;16:577-92.

6. Webb S. Historical perspective on IMRT. In: Palta JR, Mackie $T R$, Chen $Z$. Intensity-modulated radiation therapy: the state of the art. Madison, WI: Medical Physics Publishing; 2003. p. $1-23$.

7. Intensity Modulated Radiation Therapy Collaborative Working Group. Intensity-modulated radiotherapy: current status and issues of interest. Int J Radiat Oncol Biol Phys 2001;51:880914.

8. Brahme A. Optimization of stationary and moving beam radiation therapy techniques. Radiother Oncol 1988;12:12940.

9. Webb S. Optimisation of conformal radiotherapy dose distributions by simulated annealing. Phys Med Biol 1989;34:1349-70.

10. Bortfeld T, Burkelbach J, Boesecke R, Schlegel W. Methods of image reconstruction from projections applied to conformation radiotherapy. Phys Med Biol 1990;35:1423-34.

11. Ling CC, Burman C, Chui CS, et al. Conformal radiation treatment of prostate cancer using inversely-planned intensity-modulated photon beams produced with dynamic multileaf collimation. Int J Radiat Oncol Biol Phys 1996;35:721-30.

12. Spirou SV, Chui CS. A gradient inverse planning algorithm with dose-volume constraints. Med Phys 1998;25:321-33.

13. Brahme A. Optimized radiation therapy based on radiobiological objectives. Semin Radiat Oncol 1999;9:35-47.

14. Niemierko A. Reporting and analyzing dose distributions: a concept of equivalent uniform dose. Med Phys 1997;24:10310.

15. Brahme A. Multi leaf collimator (US Patent No. 4,672,212). Washington, DC: US Patent and Trademark Office; 1987.

16. Carol MP. Peacock: a system for planning and rotational delivery of intensity-modulated fields. Int J Imaging Syst Technol 1995;6:56-61.

17. Mackie TR, Holmes T, Swerdloff $S$, et al. Tomotherapy: a new concept for the delivery of dynamic conformal radiotherapy. Med Phys 1993;20:1709-19.

18. Convery DJ, Rosenbloom ME. The generation of intensitymodulated fields for conformal radiotherapy by dynamic collimation. Phys Med Biol 1992;37:1359-74.

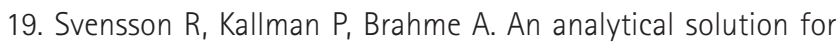
the dynamic control of multileaf collimators. Phys Med Biol 1994;39:37-61.

20. Spirou SV, Chui CS. Generation of arbitrary intensity profiles by dynamic jaws or multileaf collimators. Med Phys 1994;21:1031-41.

21. Stein J, Bortfeld T, Dorschel B, Schlegel W. Dynamic X-ray compensation for conformal radiotherapy by means of multileaf collimation. Radiother Oncol 1994;32:163-73.

22. Cho BC, Park SW, Oh DH, Bae H. Quality assurance for intensity modulated radiation therapy. J Korean Soc Ther Radiol Oncol 2001;19:275-86

23. Park SW, Oh DH, Bae HS, Cho BC, Park JH, Han SH. Application of intensity modulated radiation therapy (IMRT) in prostate cancer. J Korean Soc Ther Radiol Oncol 2002;20:68-72.

24. Shepard DM, Earl MA, Li XA, Naqvi S, Yu C. Direct aperture optimization: a turnkey solution for step-and-shoot IMRT. Med Phys 2002;29:1007-18.

25. Earl MA, Shepard DM, Naqvi S, Li XA, Yu CX. Inverse planning for intensity-modulated arc therapy using direct aperture optimization. Phys Med Biol 2003;48:1075-89.

26. Otto K. Volumetric modulated arc therapy: IMRT in a single gantry arc. Med Phys 2008;35:310-7.

27. Bichay T, Cao D, Orton CG. Point/counterpoint. Helical tomotherapy will ultimately replace linear accelerator based IMRT as the best way to deliver conformal radiotherapy. Med Phys 2008;35:1625-8.

28. Yu CX. Intensity-modulated arc therapy with dynamic multileaf collimation: an alternative to tomotherapy. Phys Med Biol 1995;40:1435-49.

29. Das IJ, Cheng CW, Chopra KL, Mitra RK, Srivastava SP, Glatstein E. Intensity-modulated radiation therapy dose 
prescription, recording, and delivery: patterns of variability among institutions and treatment planning systems. J Natl Cancer Inst 2008;100:300-7.

30. International Commission on Radiation Units and Measurements. ICRU Report 83: Prescribing, recording, and reporting photon-beam intensity-modulated radiation therapy (IMRT). J ICRU 2010;10:NP.

31. Freedman GM, Anderson PR, Li J, et al. Intensity modulated radiation therapy (IMRT) decreases acute skin toxicity for women receiving radiation for breast cancer. Am J Clin Oncol 2006;29:66-70.

32. Lee $N$, Chuang C, Quivey JM, et al. Skin toxicity due to intensity-modulated radiotherapy for head-and-neck carcinoma. Int J Radiat Oncol Biol Phys 2002;53:630-7.

33. Hall EJ, Wuu CS. Radiation-induced second cancers: the impact of 3D-CRT and IMRT. Int J Radiat Oncol Biol Phys 2003;56:83-8.

34. Wang C, Iwamoto $K_{1}$ Low D, Chen A. (S004) Trend in second malignancy risk for head and neck cancer with increased utilization of IMRT: analysis of SEER database. Oncology (Williston Park) 2016;30 Suppl:216628.

35. Journy NM, Morton LM, Kleinerman RA, Bekelman JE, Berrington de Gonzalez A. Second primary cancers after intensity-modulated vs 3-dimensional conformal radiation therapy for prostate cancer. JAMA Oncol 2016;2:1368-70.

36. Chun SG, Hu C, Choy H, et al. Impact of intensity-modulated radiation therapy technique for locally advanced non-smallcell lung cancer: a secondary analysis of the NRG Oncology RTOG 0617 randomized clinical trial. J Clin Oncol 2017;35:5662.

37. Ibbott GS, Followill DS, Molineu HA, Lowenstein JR, Alvarez $P E$, Roll JE. Challenges in credentialing institutions and participants in advanced technology multi-institutional clinical trials. Int J Radiat Oncol Biol Phys 2008;71(1 Suppl):S71-5.

38. Bogdanich W. Radiation offers new cures, and ways to do harm [Internet]. New York, NY: New York Times; 2010 [cited 2018 Mar 1]. Available from: http://www.nytimes. com/2010/01/24/health/24radiation.html.

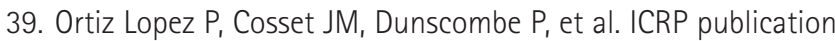

112. A report of preventing accidental exposures from new external beam radiation therapy technologies. Ann ICRP 2009;39:1-86.

40. Moran JM, Dempsey M, Eisbruch $A$, et al. Safety considerations for IMRT: executive summary. Med Phys 2011;38:5067-72.

41. Abdel-Wahab M, Rosenblatt $E_{1}$ Holmberg 0, Meghzifene A. Safety in radiation oncology: the role of international initiatives by the International Atomic Energy Agency. J Am Coll Radiol 2011;8:789-94.

42. Hoopes DJ, Dicker AP, Eads NL, et al. RO-ILS: Radiation Oncology Incident Learning System: a report from the first year of experience. Pract Radiat Oncol 2015;5:312-8.

43. Siochi RA, Molineu A, Orton CG. Patient-specific QA for IMRT should be performed using software rather than hardware methods. Med Phys 2013;40:070601.

44. Pawlicki T, Yoo S, Court LE, et al. Moving from IMRT OA measurements toward independent computer calculations using control charts. Radiother Oncol 2008;89:330-7.

45. Monz M, Kufer KH, Bortfeld TR, Thieke C. Pareto navigation: algorithmic foundation of interactive multi-criteria IMRT planning. Phys Med Biol 2008;53:985-98.

46. Ling CC, Humm J, Larson S, et al. Towards multidimensional radiotherapy (MD-CRT): biological imaging and biological conformality. Int J Radiat Oncol Biol Phys 2000;47:551-60.

47. Gutierrez AN, Westerly DC, Tome WA, et al. Whole brain radiotherapy with hippocampal avoidance and simultaneously integrated brain metastases boost: a planning study. Int J Radiat Oncol Biol Phys 2007;69:589-97.

48. Boyer AL, Desobry GE, Wells NH. Potential and limitations of invariant kernel conformal therapy. Med Phys 1991;18:70312.

49. Bortfeld TR, Kahler DL, Waldron TJ, Boyer AL. X-ray field compensation with multileaf collimators. Int J Radiat Oncol Biol Phys 1994;28:723-30.

50. Carol M, Grant WH 3rd, Pavord D, et al. Initial clinical experience with the Peacock intensity modulation of a 3-D conformal radiation therapy system. Stereotact Funct Neurosurg 1996;66:30-4. 